\title{
Design of high power LED-based UVA emission system and a photosensitive substance for clinical application in corneal radiation
}

Alessandro D. Mota, André M. Cestari, André O. de
Oliveira, Anselmo G. Oliveira, Cristina H. B. Terruggi, et al.

Alessandro D. Mota, André M. Cestari, André O. de Oliveira, Anselmo G. Oliveira, Cristina H. B. Terruggi, Giuliano Rossi, Jarbas C. Castro, João P. B. Ligabô, Tiago A. Ortega, Tiago Rosa, "Design of high power LED-based UVA emission system and a photosensitive substance for clinical application in corneal radiation," Proc. SPIE 9571, Fourteenth International Conference on Solid State Lighting and LED-based Illumination Systems, 95710X (8 September 2015); doi: 10.1117/12.2187265

Event: SPIE Optical Engineering + Applications, 2015, San Diego, California, United States 


\title{
Design of high power LED-based UVA emission system and a photosensitive substance for clinical application in corneal radiation
}

\author{
Alessandro D. Mota ${ }^{* 1}$, André M. Cestari ${ }^{1}$, André O. de Oliveira ${ }^{1,2}$, Anselmo G. Oliveira ${ }^{3}$, Cristina H. \\ B. Terruggi ${ }^{3}$, Giuliano Rossi ${ }^{1}$, Jarbas C. Castro ${ }^{2}$, João P. B. Ligabô ${ }^{1}$, Tiago A. Ortega ${ }^{1}$, Tiago Rosa ${ }^{1}$ \\ ${ }^{1}$ Opto Eletrônica S/A, 1071 Joaquim A. R. de Souza, São Carlos, SP, Brazil 13560-330; \\ ${ }^{2}$ Instituto de Física de São Carlos, Universidade de São Paulo, 400 Trabalhador São Carlense, São \\ Carlos, SP, Brazil 13560-970 ; \\ ${ }^{3}$ Faculdade de Ciências Famacêuticas, Universidade Estadual Paulista, km1 Rodovia Araraquara - \\ Jaú, Araraquara, SP, Brazil 14801-902
}

\begin{abstract}
This work presents an innovative cross-linking procedure to keratoconus treatment, a corneal disease. It includes the development of an ultraviolet controlled emission portable device based on LED source and a new formulation of a photosensitive drug called riboflavin. This new formulation improves drug administration by its transepithelial property. The UV reaction with riboflavin in corneal tissue leads to a modification of corneal collagen fibers, turning them more rigid and denser, and consequently restraining the advance of the disease. We present the control procedures to maintain UV output power stable up to $45 \mathrm{mw} / \mathrm{cm}^{2}$, the optical architecture that leads to a homogeneous UV spot and the new formulation of Riboflavin.
\end{abstract}

Keywords: Ultraviolet LED, Cross-linking, Keratoconus, Riboflavin, Transepithelial, PID control system for LEDs, Close loop system for LED, UV based Optical system.

\section{INTRODUCTION}

Corneal collagen cross-linking (CXL) has introduced a promising alternative to Keratoconus treatment ${ }^{1}$. The keratoconus is an ectatic, non-inflammatory, progressive disease that causes corneal tissue structure weakening, leading to a conical shaped cornea, therefore loss of visual acuity (progressive myopic visual impairment and irregular astigmatism). Statistically, the incidence of this disease is between 20 and 230 per $100.000^{2}$. In recent years, cross-linking has been considered an alternative method to keratoconus stagnation, and can avoid cornea transplantation or introduction of contact lenses. Wollensak et al. have established the conventional treatment protocol, which is divided in two stages: Riboflavin administration and UV light exposure. This technique consists in administration of photosensitive substance called Riboflavin (solution of vitamin B2) to enhance the UV absorption in the cornea. The application of riboflavin drops is done every two minutes for 30 minutes (pre-soak time). The epithelium, membrane that covers the cornea, is removed in order to allow penetration of Riboflavin into the cornea in a region called stroma. After this, cornea is subjected to a low power density of UV, $3 \mathrm{~mW} / \mathrm{cm}^{2}$ for 30 minutes, resulting in a total dose of $5.4 \mathrm{~J} / \mathrm{cm}^{2}$. Interaction between riboflavin and UV light induces the formation of more bonds among adjacent collagen molecules, which modifies corneal structure by making it more rigid and denser. Therefore, the result is a less susceptible cornea to shape modification, avoiding the keratoconus advance.

Recently, the procedure to reduce the time of treatment by increasing the power density on corneal tissue, called accelerated procedure, has been investigated by doctors. $\mathrm{Chew}^{3}$ presented that the same clinical results can be achieved by reducing the total time of UV exposure by increasing the power density to match with well-known energy dose of 5.4 $\mathrm{J} / \mathrm{cm}^{2}$. He got reports of doctors who are practicing the accelerated procedure with their patients, and they are confident that this technique is worthy and efficient. Ronald R. Krueger ${ }^{4}$ has shown that short time treatment can provide same corneal strengthening as lower powers over longer time periods, or there is no statistically significant biomechanical difference in both cases. John Mashal ${ }^{5}$, conducted an experiment of UV exposure to measure the effects on human endothelium cells, the deeper membrane in corneal tissue, which has to be preserved. He showed that viability of the cells did not show significant difference between low or high irradiance and was not cytotoxic to human endothelium

Fourteenth International Conference on Solid State Lighting and LED-based Illumination Systems edited by Matthew H. Kane, Jianzhong Jiao, Nikolaus Dietz, Jian-Jang Huang, Proc. of SPIE Vol.

9571, 95710X · C 2015 SPIE · CCC code: 0277-786X/15/\$18 · doi: 10.1117/12.2187265 
cells. Additionally, Sliney ${ }^{6}$ made a study of safety in accelerated cross-linking procedure offered by Avedro ${ }^{7}$ relating corneal absorption dose of UV with depth of corneal tissue. In accelerated procedure, the pre-soak time duration, time that corneal is under Riboflavin presence before UV exposure, is shorter than conventional procedure, resulting in quite same dose of UV absorption in region nearby endothelium. It leads to conclusion that accelerated procedure is as safe as the conventional procedure.

The equipment presented in this work was developed to attend the standard and accelerated well-known established protocols applied in CXL Keratoconus treatment. It is a portable type A ultraviolet emission device with peak wavelength at $365 \mathrm{~nm}$ and output power density up to $45 \mathrm{~mW} / \mathrm{cm}^{2}$. This power let the procedure duration shorter, and can be theoretically performed in 2 minutes. It is composed of a UV illumination source attached to a special set of lenses and mirrors to project the light on the patient's cornea. Such light projection has uniform power distribution and homogeneous light density in the illuminated area. In addition, a red light source is coupled into the system to indicate to the doctor the focus of the optical system. An electronic system will be responsible to control light emission and is composed of a microcontroller circuit connected to a power control loop with feedback and a current driver. The critical point of this system is to maintain a constant light output for treatment and achieve a high level of optical efficiency capable to provide $45 \mathrm{~mW} / \mathrm{cm}^{2}$ at the output. This system results in constant output power and in uniform power distribution, with less than $10 \%$ of variation in both cases.

Additionally, Anselmo Oliveira presents new studies intending to eliminate the necessity to remove the corneal epithelium to permit Riboflavin penetration in cornea. He proposes the development of a solution that consists in the incorporation of the hydrophilic riboflavin in a system with nanometric dimensions, as the nanoemulsion, which combines the properties of bioadhesion and permeability increasing, to allow the riboflavin permeates through the epidermis of the cornea and fixes in stroma. Although he presents good results, the pre-soak time duration was too long for treatment purpose. A new formulation of the nanostrctured riboflavin was created and is presented in this work, and the main goal is to make possible the reduction of penetration time duration in CLX treatment procedure.

Finally, this work is divided as follows: section 1 is the introduction; the UV LED system device and the nanoemulsion of riboflavin are in section 2; the results obtained with the created control system and the formulation of new riboflavin are in section 3; conclusions are in section 4.

\section{METHODOLOGY}

\subsection{Accelerated Cross-linking}

The technique proposed by this work is already being applied by international companies such as Avedro ${ }^{7}$ and doctors as Ronald R. Krueger ${ }^{4}$. Accelerated cross-linking consists in reducing the time of ultraviolet radiation application by increasing the power dose and keeping the same total treatment energy $\left(5.4 \mathrm{~J} / \mathrm{cm}^{2}\right)$. The UV emission device developed in this work has output power enough to reduce the application time up to 2 minutes by setting output power to 45 $\mathrm{mW} / \mathrm{cm}^{2}$. The procedure consists in three steps. First, scrapes or flaps are made on corneal epithelium to permit riboflavin (photosensitive solution of vitamin B12) to penetrate in internal corneal tissue called stroma. Figure 1 shows the membranes involved on treatment. After some days the epithelium is naturally recomposed. Second, drops of riboflavin are dispensed on corneal surface every 5 minutes during a period of 10 to 30 minutes (time dependent of UV power). This time is called pre-soak time, which is suggested by recent researches to be of 10 minutes using a power of $30 \mathrm{~mW} / \mathrm{cm}^{2}{ }^{8-10}$. Third, the cornea is exposed to ultraviolet light (4 minutes irradiation with $30 \mathrm{~mW} / \mathrm{cm}^{2}, 365 \mathrm{~nm}$ UVA). Additionally, there are no treatment tests with a higher dose, but it is supposed that doctors will come up with new studies using shorter time application, as the new machine developed in this work is able to offer higher power. Furthermore, the procedure will use a new formulation of riboflavin that is based in nanoemulsion platform (riboflavin5-phosphate), which does not demand removal of the corneal epithelium during pre-soak time. This application is called transepithelial. The advantages of using the accelerated cross-linking in junction with the transepithelial riboflavin are as

follow: fast procedure, most patients can be treated; low risk of infection because of epithelium is not removed; painless procedure; quick recover of the patient; the procedure can be performed out of operating room because there is no surgical intervention (low treatment cost). 
Figure 2 presents the simulation of UV procedure. Figure 2-A shows the corneal flaccid shape and weak bonds in collagen fibers of the cornea; Figure 2-B shows the pre-soak procedure, in which drops of riboflavin are dispensed on corneal tissue; Figure 2-C shows the UV light emission on corneal surface, which is responsible to create more bonds in collagen fibers and make corneal tissue more rigid; Figure 2-D shows the result of treatment, more bonds were created in cornea tissue, it is more rigid and denser, thus is less susceptible of shape changes (it avoids the advance of keratoconus).

Figure 1. Corneal tissue structure ${ }^{11}$.
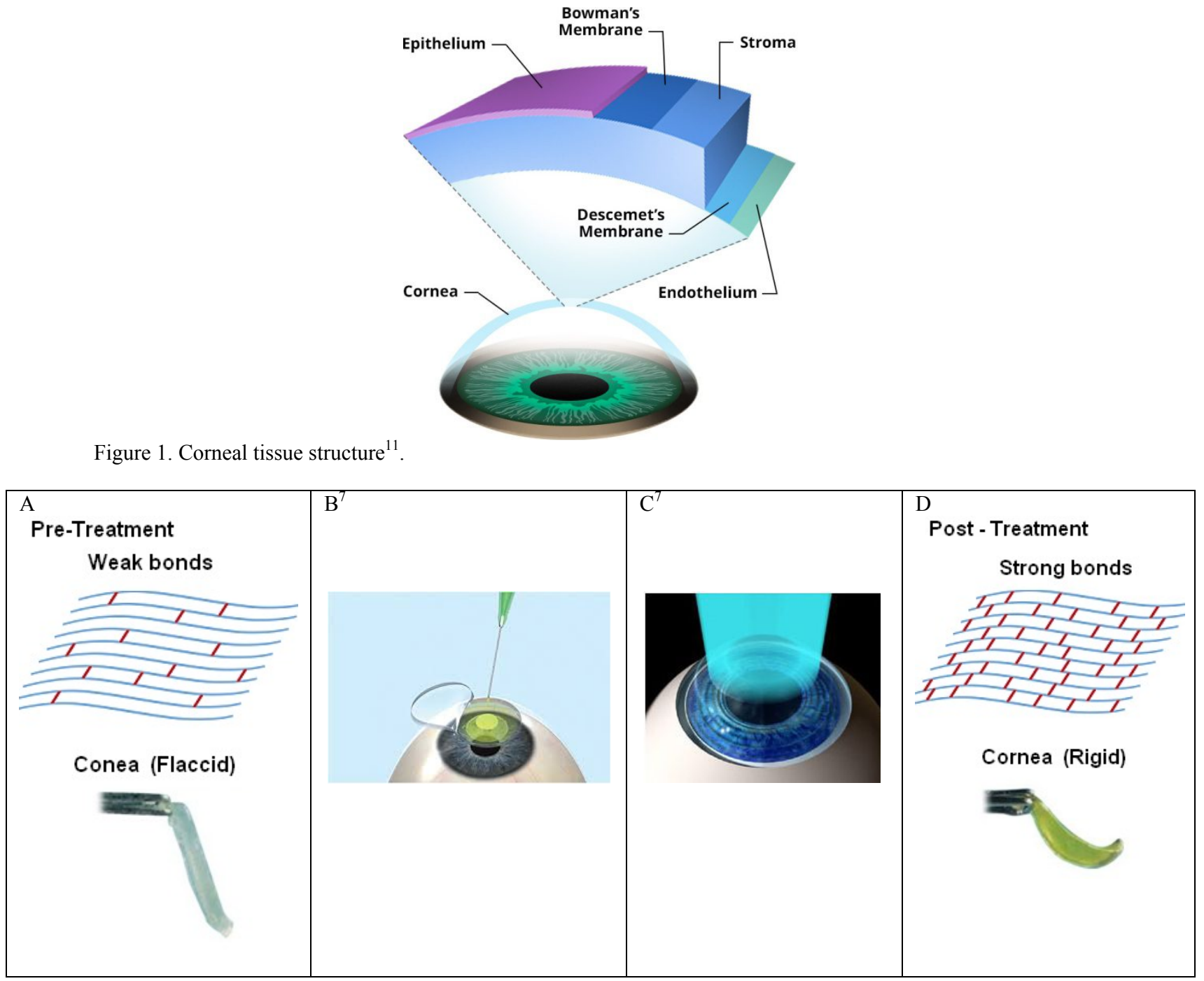

Figure 2. Illustration of cross-linking procedure. A - Cornea state before UV radiation, presents weak bonds in collagen fibers, flaccid shape; B - Epithelium removal and drops of riboflavin disposal; C - Application of UV homogenous radiation; D - Cornea state after treatment, presents stronger bonds in collagen fibers and a rigid shape.

\subsection{Optics System}

As formerly discussed, treatment requires a homogenized beam to be delivered in order to avoid hot spots which may lead to localized sub-threshold irradiation, causing some areas to become more flaccid then others, which may lead to development of a localized Keratoconus. An UV illumination system was designed based on LED as showed in Figure 3. Light from an UV LED with peak wavelength at $365 \mathrm{~nm}$ is focused first at surface 1, where a diaphragm is placed to 
limit rays that go through it. This diaphragm controls beam size delivered at focal plane, illuminating patient's eye with top-hat beam of 6,8 and 10 millimeters of diameter. As the user changes the aperture of the iris, power delivered by the LED is electronically balanced to maintain a constant power density at focal plane. Figure 4 shows the simulated power distribution on focal plane for beam size of $10 \mathrm{~mm}$.

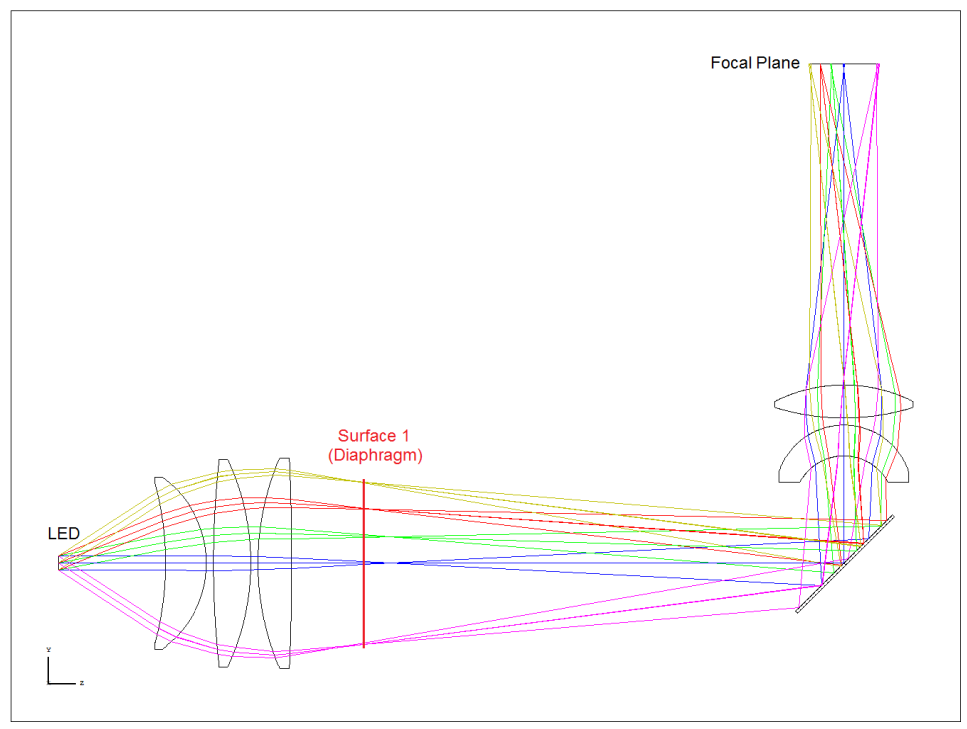

Figure 3. 3D Layout of optical design (obtained from ZEMAX®).

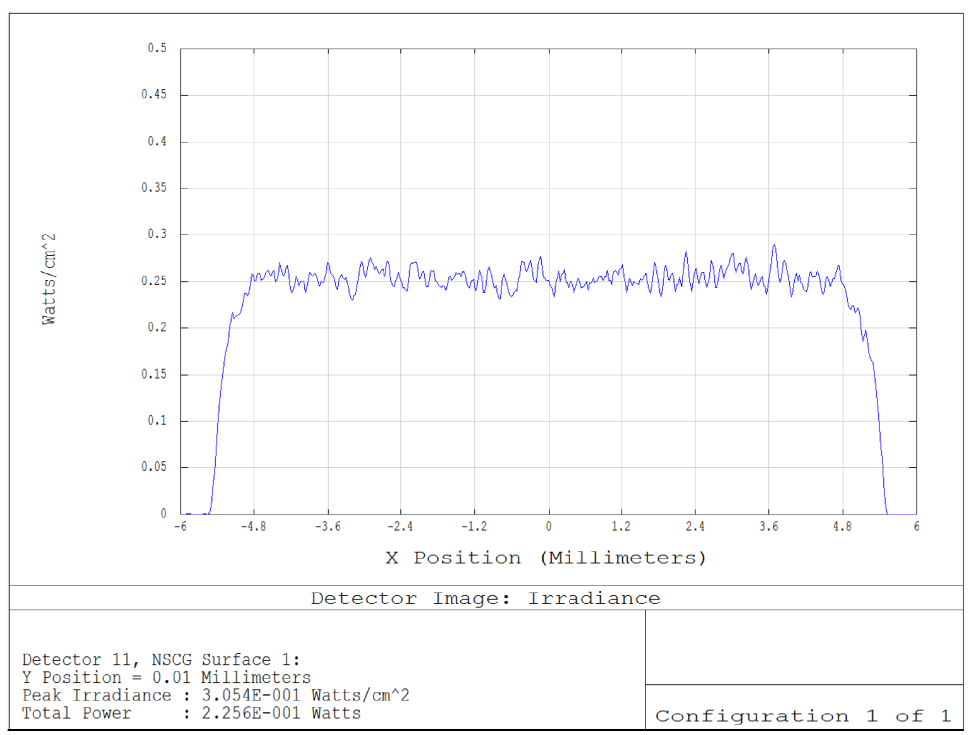

Figure 4. Focal plane simulation (obtained from ZEMAX®).

\subsection{LED power Control system}

Figure 5 shows the block diagram of the entire electronic control system. The opto-electronic equipment consists of one UV LED source $(365 \mathrm{~nm})$, one optical system projection that carries the UV light to patient's cornea with homogeneous power distribution and a microcontrolled electronic system for output power and time duration control. The controller is a system which aims to generate a desired output signal from a reference signal at the input. It is defined as a causal dynamic system, since the output depends not only on entry into the present moment, but also on the entry at the previous instant. There are two types of controllers: open and closed loop system. In the open loop system, the controller is basically an actuator using the reference for the desired response. The output does not affect the input reference. On 
the other hand, the closed loop system uses sensors for measuring and adjusting the current output by changing the reference value to obtain the desired output. The sample of output signal is called feedback ${ }^{12}$. The controller used in this work is a closed loop system called PI (Proportional - integrative). The proportional gain allows signal stability for high frequency responses. This ensures that in fast response, a short rise time (milliseconds), the system remains stable. The integrative term guarantees that the average error (steady-state error) is close to zero, or the difference between the reference signal and the desired output tends to zero ${ }^{13}$. The presented control system has two PIs, PI1 in software, and PI2 in hardware, and they are connected in a cascade configuration. The current sensor (precision resistor), LED current driver, which is based on Mosfet current source ${ }^{14}$, and PI2 are part of the current control loop. It guarantees that the LED current is auto-adjusted proportionally to the difference of current reference signal in the control loop input and the instantaneous current read by precision resistor. The photodiode and microcontroller with PI1 are part of power control loop. PI1 keeps output power constant by changing the current reference in PI2 input proportionally to any variation of output power read from photodiode. It avoids output power fluctuations due to non-linearity effects in LED or any loss of irradiance efficiency during its lifetime. The result of this topology is a linear and proportional relation between the power references from the microcontroller to the output power of the optical system. The PI1 digital controller obeys the sequence of equations (1), (2), (3), and (4), which are programmed in an eight bits microcontroller. It is divided in calculating the error between set power and the read power in (1), calculating the proportional portion of the controller in (2), calculating the integrative portion in (3) and summing the two responses to obtain the current reference, RefPot, to load the PI in hardware in (4). The performance of the controller is calibrated by $K p$ and $K i$ constants to operate in a stable state (no oscillations), without overshoot, minimum steady error, and fast responses (short rise time) ${ }^{12}$. The graphic on Figure 5 illustrate these signal quality parameters $(\mathrm{Y}(\mathrm{t})$ is normalized to output steady-state power). It is important to note that it was not necessary to use the derivative term of PID, as this term is responsible to enlarge the frequency response of the controller, and LED does not demand large frequency bandwidth.

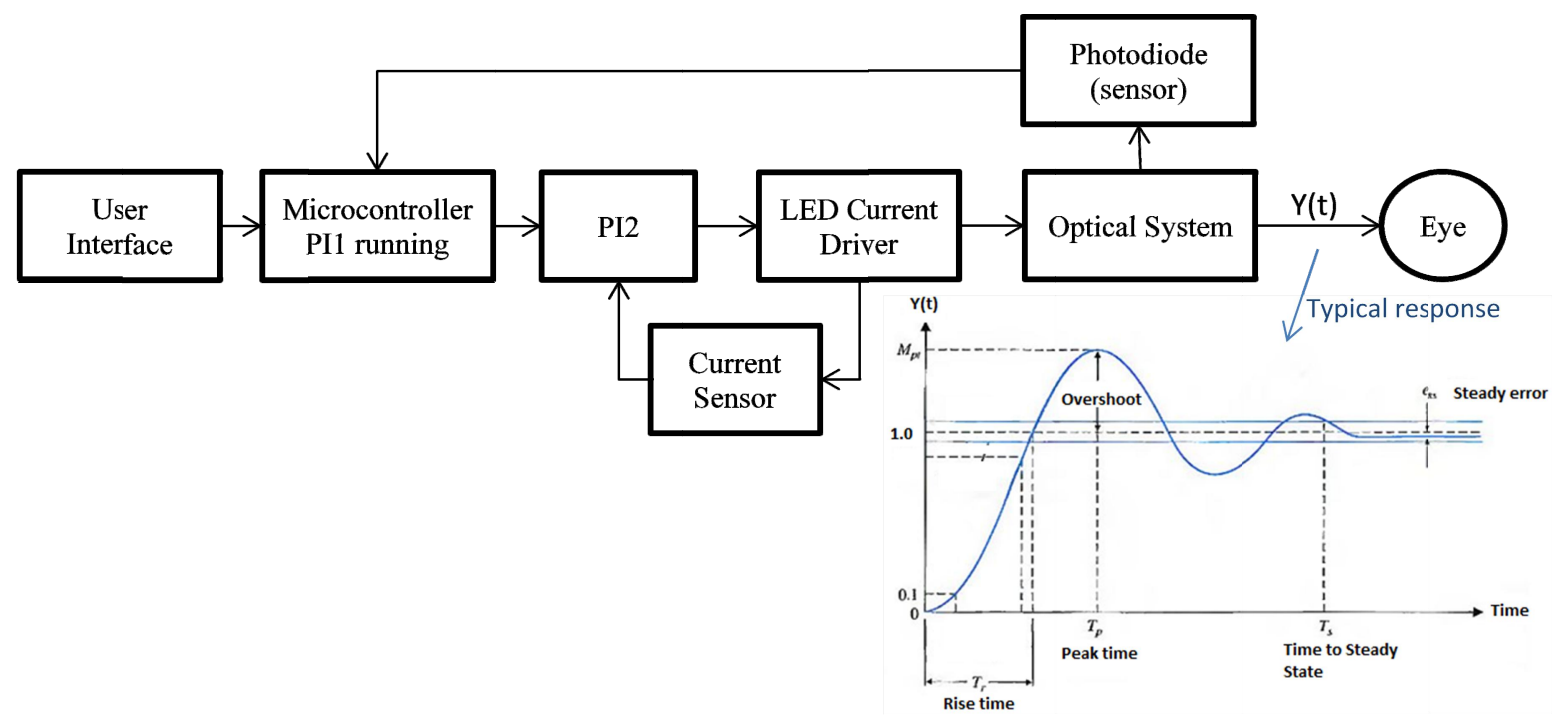

Figure 5. Block Diagram of Control Loop System.

$$
\begin{aligned}
& E r r=P o t-\text { Pot }_{365} \\
& \operatorname{Prop}=E r r * K p \\
& \operatorname{Int}_{1}=E r r * K i+I n t_{0} \\
& \operatorname{Ref}_{\text {Pot }}=\text { Prop }+ \text { Int }_{1}
\end{aligned}
$$

For equations (1) to (3), Err is the difference between output power set and read, Pot is the output power set, Pot $_{365}$ is the LED output power read, Prop is the proportional term of controller PI, $K p$ is the proportional constant of PI, Int $_{1}$ is 
current integrative term of PI, $K i$ is integrative constant of PI, Int $t_{0}$ is the integrative term of the last interaction, and $R_{e f} f_{P o t}$ is the resulting current reference ${ }^{15}$.

\subsection{Riboflavin-Nanostructured}

The standard protocol of CXL proposed by Wollensak et al. ${ }^{1}$ recommends removing the corneal epithelium before treatment to allow the penetration of riboflavin into the stroma. Experimental and clinical research has shown that the intact epithelium does not block the effects of ultraviolet light (UVA) ${ }^{16}$, but reduces the effectiveness of treatment by altering adequate diffusion of riboflavin into the stroma ${ }^{17}$. However, epithelium removal causes risks and can increase the frequency of infections in the cornea, opacity, scars and infiltration ${ }^{18-20}$. Moreover, it can cause pain, photophobia and delayed visual rehabilitation. In a previous project, Anselmo et al. evaluated the stromal penetration of riboflavin phosphate in nanoemulsion biocompatible formulation, using intact rabbit corneas. Although the absorption of nanoemulsion riboflavin phosphate was similar to the standard technique of cross-linking with epithelium removal, the time of penetration was too large, around 120 minutes $^{21}$.

Among technological possibilities, pharmaceutical formulations based on nanotechnology platform constitute an innovative way to facilitate penetration of riboflavin through the corneal epithelium. The standard riboflavin solution (10

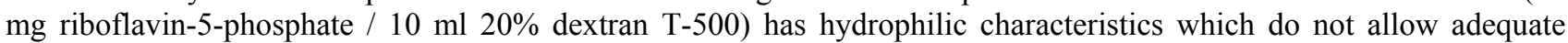
diffusion through the corneal epithelium. Considering the physico-chemical properties of riboflavin base and riboflavin phosphate, they do not have the ability to permeate into the stroma. Due to lipophilic characteristic of corneal epithelium, riboflavin base interacts with the corneal epithelium and do not properly permeates into the stroma. Moreover, due to the riboflavin phosphate characteristic of hydrophilicity and anionic character, it is repelled from the surface of the cornea and riboflavin do not sufficiently permeate into the stroma. Thus, a technologically feasible delivery system involves incorporation of the hydrophilic riboflavin in a system with nanometric dimensions, as the nanoemulsion, which combines the properties of bioadhesion and permeability increasing, so as to allow the riboflavin to permeate through the corneal epidermis (lipophilic) and secure the stroma (hydrophilic). Thus, the bioadhesive property of the vehicle will allow the hydrophilic riboflavin have an intense contact with the surface of the cornea and increase the permeability of riboflavin. It will allow the passage through corneal tissue, with retention in the stroma, as the corneal endothelium also has lipophilic character. The main goal with this new formulation is to achieve proper concentration of riboflavin in stroma by not demanding hours of pre-soak time.

\section{RESULTS}

\subsection{Control system}

The embedded electronic was developed and assembled with all the principles shown earlier (Figure 5) in the UV LED console in order to evaluate the performance of the optics and control system. The embedded control software was developed in the same way as shown in equation 1 to 4 . To evaluate the control system process, the LED control system was turned on and output power and LED current wave forms were collected by an oscilloscope. It is possible to analyze the behavior of the LED current and light output power during the transitory and steady-state regime and verify if the control system response time is capable to produce rise time of milliseconds and no oscillations. If the rise-time of the pulse is too long when compared to the pulse duration, the delivery energy will be reduced to values that will not produce the desired therapeutic effects. Due to cross-linking procedure establishment pulse duration of 30 minutes, or accelerated going to 10 minutes, rise time response in milliseconds is fast enough to do not compromise total energy density of treatment.

Figure 6 shows three different power responses of the LED control system. Chanel 1 of oscilloscope is the LED current read by precision resistor sensor, and channel 2 is the LED power output read by photodiode. The current relation in the sensor circuit is $23 \mathrm{~V} / 1 \mathrm{~A}$, and the power relation in photodiode circuit is $0.133 \mathrm{~V} / 1 \mathrm{~mW}$. Graph $A$ is the power response to a set point value of $5 \mathrm{~mW}$, graph $B$ is the power response to a set point value of $15 \mathrm{~mW}$, and Graph $C$ is the power response to a set point value of $36 \mathrm{~mW}$. The responses are stable, no overshoot (current obeyed a smooth crescent ramp in the initial process), there is short rise time, and less than $10 \%$ of steady-state error. This parameter is important because the LED device is a medical equipment, and is supposed to attend IEC 60601-1 and IEC 60602-2-22 standards, which limit LED power output variations up to $20 \%$. 


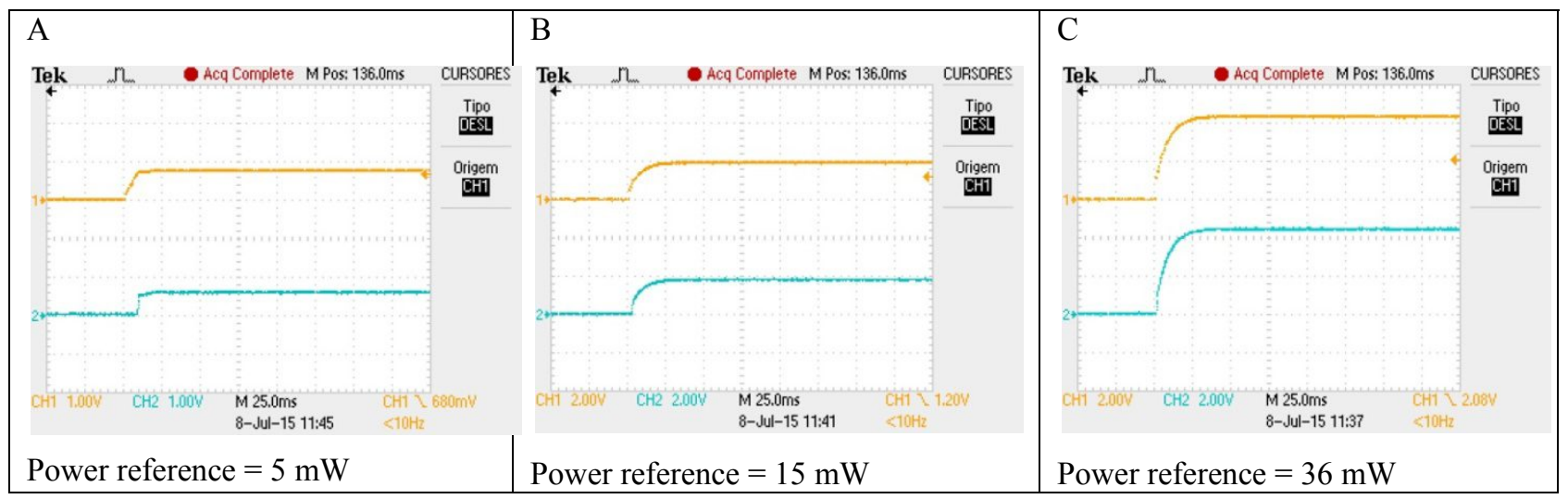

Figure 6. Laboratory power and current behavior of LED. Stored in oscilloscope Tektroniks TDS2024B.

Table 1 presents the final LED control system calibration performance, listing main parameters in different output power setting. The measures cover the output power range of the UV LED device. It is clear the linearity of set power to the output power, even though the LED current to power output is non-linear. It proves the effect of the power control loop system. Figure 7 shows the output power by LED current. Maximum power was set to $36 \mathrm{~mW}$ because it corresponds to $45 \mathrm{~mW} / \mathrm{cm}^{2}$ with a beam diameter of $10 \mathrm{~mm}$. One interesting point is that the maximum power was achieved by running the LED with $190 \mathrm{~mA}$. It is lower than the recommended work current of $500 \mathrm{~mA}$ on datasheet ${ }^{22}$. Then, if new researches demand more power, this device is ready to provide.

Table 1. Output power performance of UV LED control system. Power was measured by the power meter Coherent FiledMaxII TO and power head model PS19Q.

\begin{tabular}{|c|c|c|c|c|}
\hline Power Set (mW) & Power Read (mW) & Steady-State error (\%) & Rise-time (ms) & LED Current (mA) \\
\hline 1.00 & 1.00 & 0 & 10 & 26,96 \\
\hline 5.00 & 4.90 & 2.04 & 15 & 34.78 \\
\hline 15.00 & 15.60 & 3.85 & 25 & 86.96 \\
\hline 25.00 & 26.60 & 5.30 & 28 & 130.45 \\
\hline 36.00 & 38.00 & 5.26 & 30 & 187.84 \\
\hline
\end{tabular}

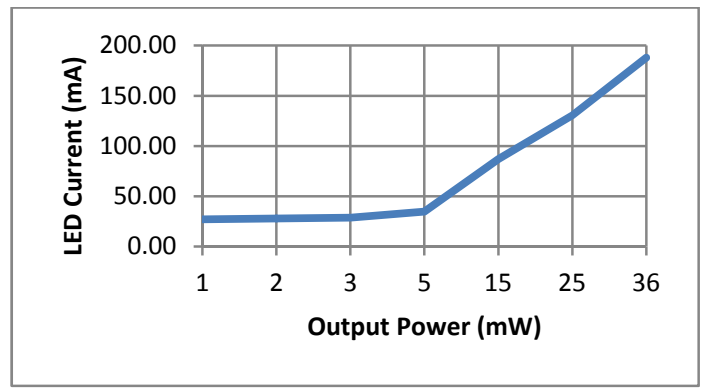

Figure 7. LED current versus Cross-linking device output power.

\subsection{Optical system}

The LED radiant flux at maximum output power of cross-linking device, for $187.84 \mathrm{~mA}$, is $169 \mathrm{~mW}(0.9 \mathrm{~mW} / \mathrm{mA})$, data from LED datasheet ${ }^{22}$. It enables to measure the system efficiency (output flux/input flux) taking in account the power losses in the optical system, LED divergence and Joule effect in LED semiconductor. If the system delivers $36 \mathrm{~mW} @$, $187.84 \mathrm{~mA}$, and LED is emitting $169 \mathrm{~mW}$, the efficiency is $21 \%$, which is not an issue, as the LED works in a light current regime to obtain the desired output power. 
Beam shape at focus plane is the most important characteristic imposed by the optical system. It has to present a homogeneous power distribution at the illuminated area to create an equal cross-linking effect at the entire corneal tissue. Figure 8, Figure 9 and Figure 10 present power distribution at focus plane got by beam analyzer Beamage Focus 1 of GentecEO. They show that beam area has less than $\pm 10 \%$ of power variations at average line of beam power. This work considered the standard applied in lasers (IEC 60601-2-22) for approval beam homogeneity, because it is also applied to high power LED medical devices. Variation less than $\pm 20 \%$ of average power is acceptable to lasers devices. There are some not continuous points at image because of dead pixels on CCD sensor.

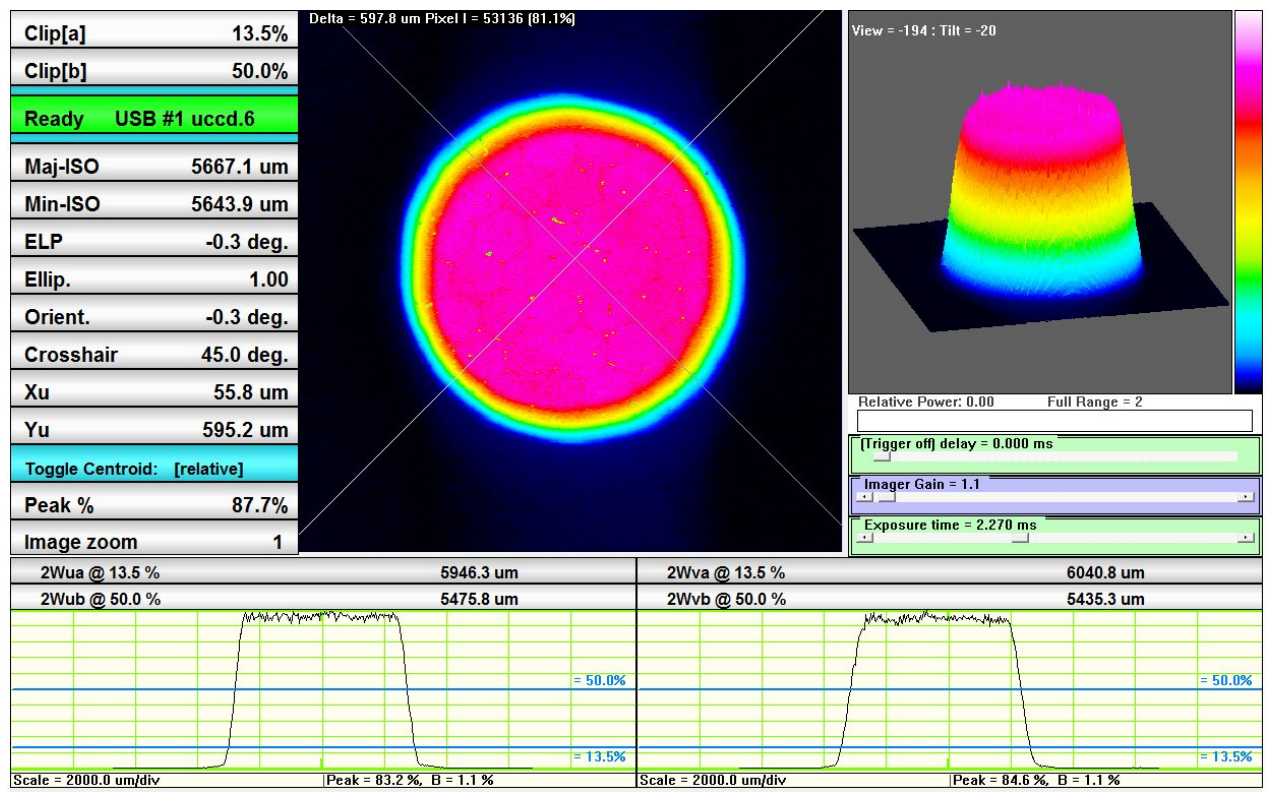

Figure 8. UV beam shape at focal plane, spot of $6 \mathrm{~mm}$.

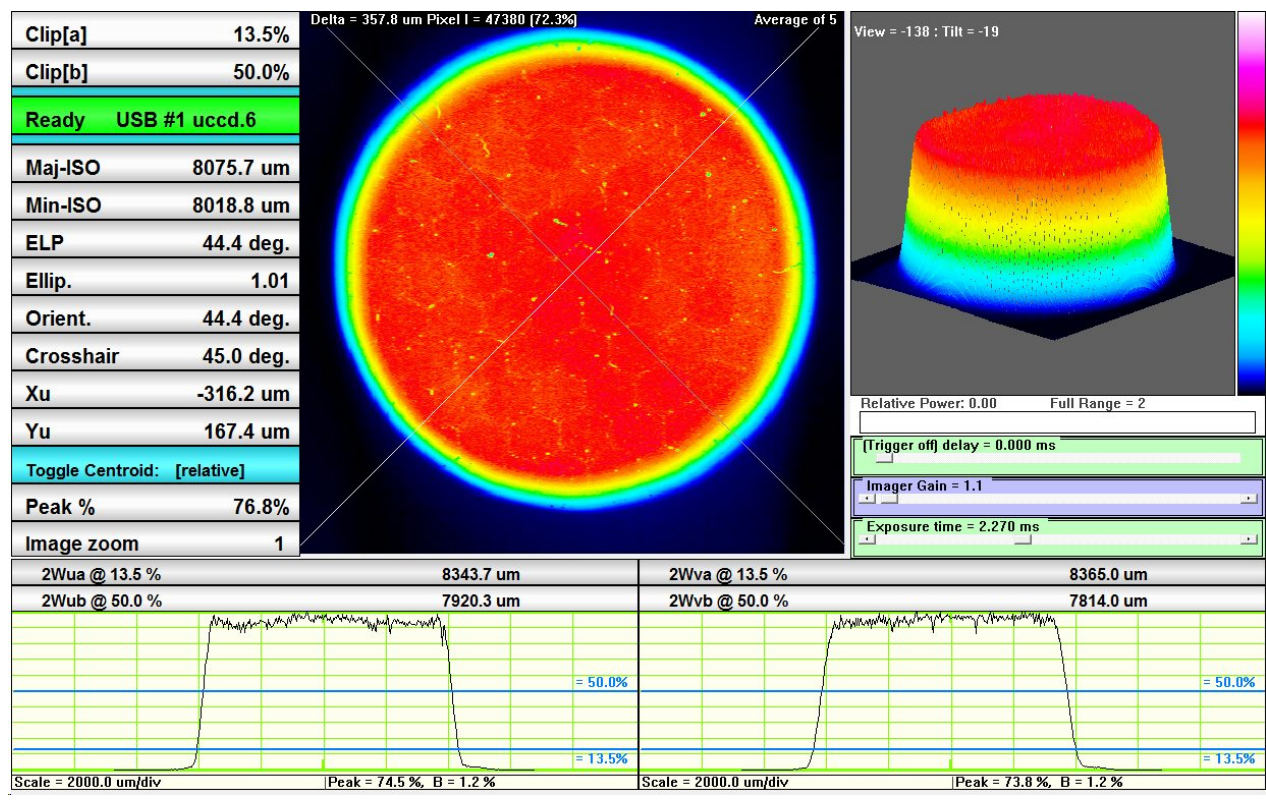

Figure 9. UV beam shape at focal plane, spot of $8 \mathrm{~mm}$. 


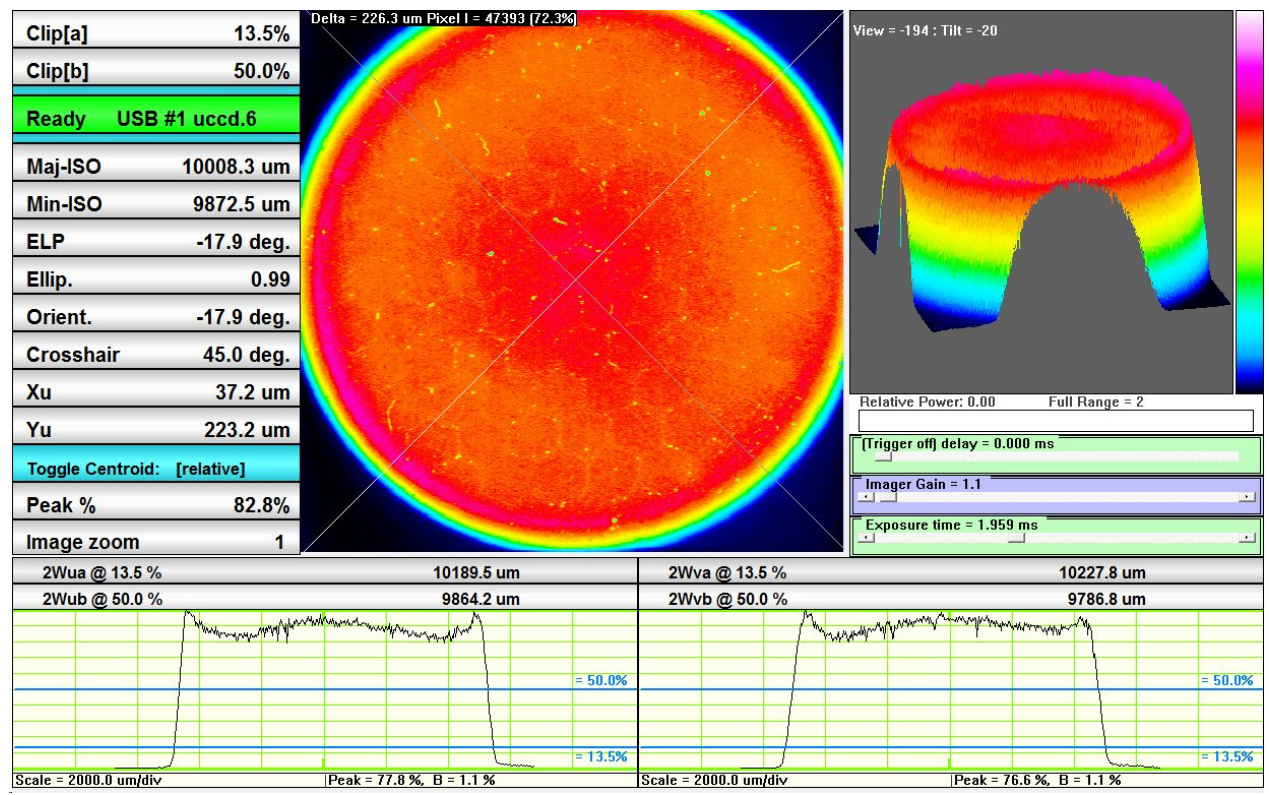

Figure 10. UV beam shape at focal plane, spot of $10 \mathrm{~mm}$.

\subsection{Cross-linking device}

Figure 11 shows a picture of the Cross-linking console assembly. The optical system is placed inside a 3D plastic prototype, all optics are mounted inside machined aluminium mounts, electronic circuit and display are connected out of the console prototype. All tests were done using this assembly, which will pass through engineering process to turn into a final product to be manufactured soon by Opto Eletronica SA. Figure 12 shows the optical system of UV light and the red color crosshair aiming beam to guide doctor in aiming the beam on patient's cornea. Figure 13 shows the design of the device that will be produced and its commercial name is Opto XLink.

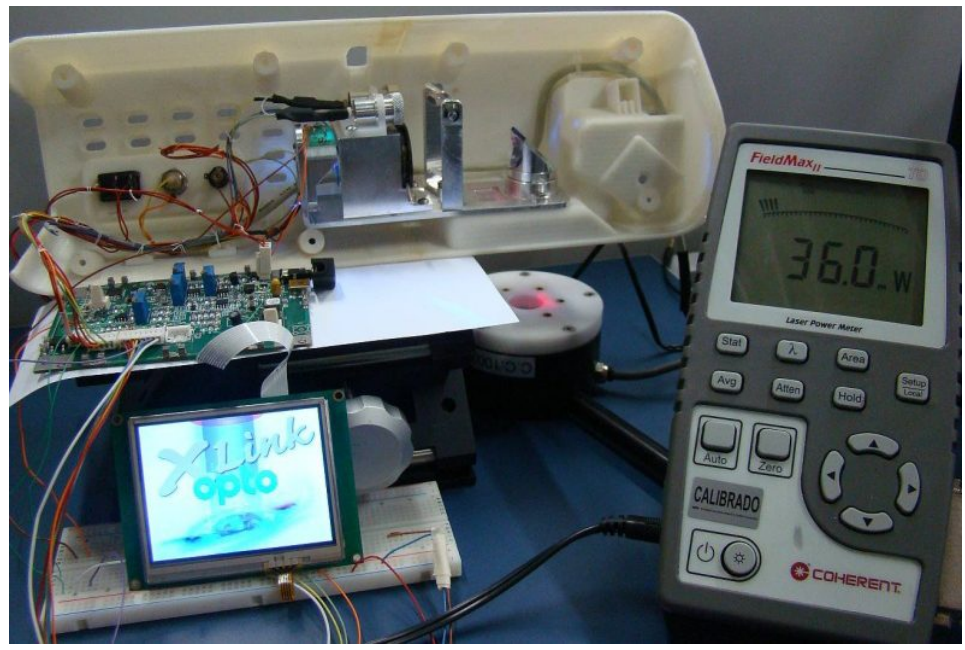

Figure 11. Cross-linking device assembly. 


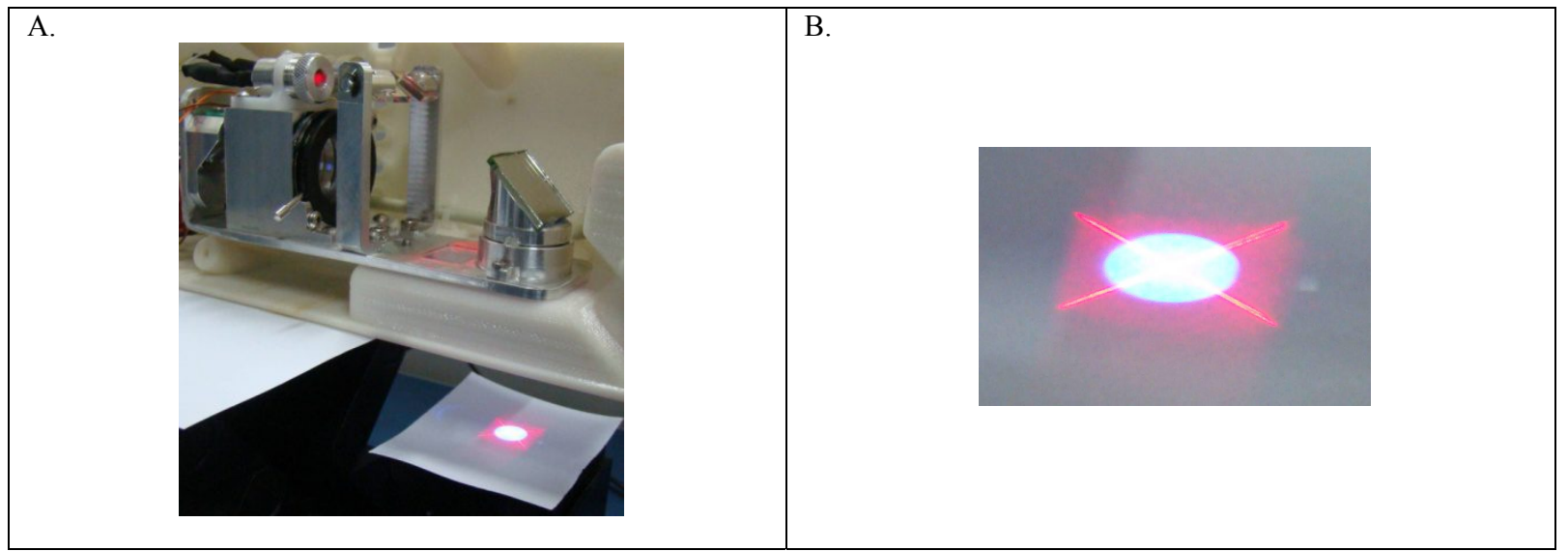

Figure 12. A - Prototype of the UV optical system. B - Details of the focal plane of aiming beam and UV light.

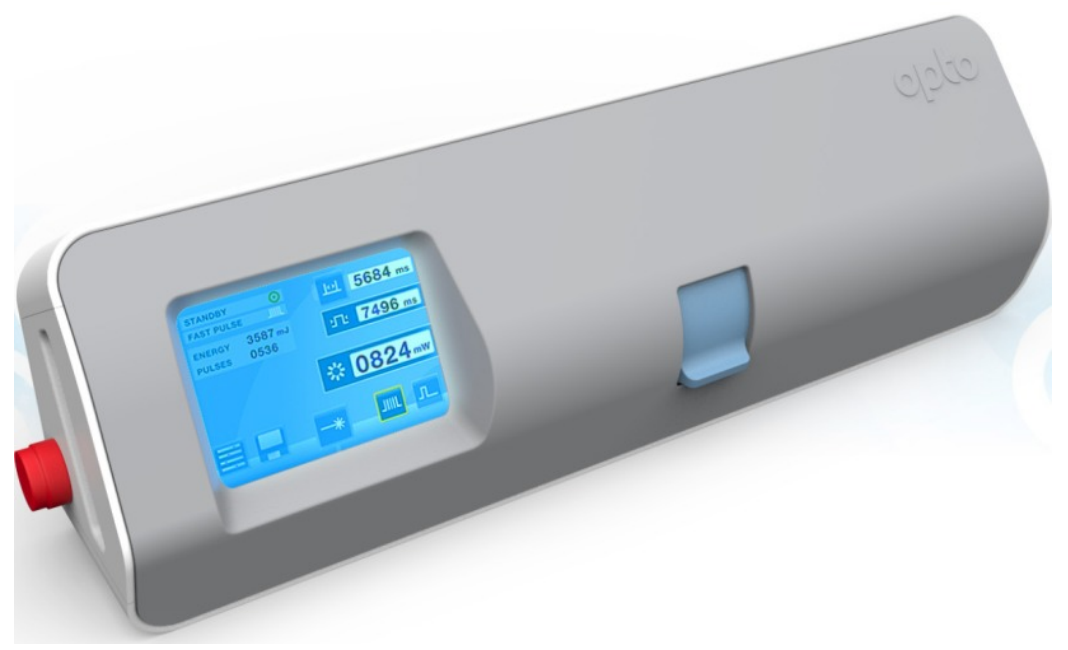

Figure 13. Opto XLink prototype design.

\subsection{Riboflavin}

The base formulation contains riboflavin phosphate $1 \%$, soy phosphatidylcholine, nonionic surfactant, cationic surfactant, block copolymer and saline (Table 2). For the systems preparation, the samples were irradiated with ultrasound, in sonication tubes for 12 minutes in a batch regime with an irradiation time of 1 minute and 30 seconds, using amplitude 8. The determination of the hydrodynamic diameter and zeta potential were performed using the Zetasizer (Malvern Instruments) equipment ${ }^{23}$. Apparent viscosity measurements were performed with a rheometer ${ }^{24}$.

Regardless of the ratio of lipid, the nanoemulsion showed rheological profile material and thixotropic non-Newtonian, but the viscosity decreases with shear rate. However, the relaxation of the shear rate leads to a viscosity recovery time dependent, which provides a superior viscosity profile at shear return to the initial situation. It was also found that increasing the proportion of the lipid in nanoemulsion resulted viscosity values higher than the lowest concentration (Figure 14), although it has not caused changes in the rheological. The maximum values for the zeta potential were about 32.5 and $36.5 \mathrm{mV}$ (Table 3), and constant concentration of the block copolymer shows excellent stability of the colloidal dispersion. However, these values decrease to about $19 \mathrm{mV}$ by increasing copolymer concentration, and it demonstrates that the PLU adhered on the surface of oil phase droplets, leaving their polyethylene chains exposed on the surface, causing an impediment to the approach of scattered ions in the medium and decreasing the reading surface potential (Table 3). 
Table 2. Formulation of nanoemulsion of riboflavin phosphate

\begin{tabular}{|l|l|}
\hline Constituent & Proportion \\
\hline Soy phosphatidylcholine (SPC) & $12 \%$ \\
\hline Medium chain triglyceride (MCT) & $5-10 \%$ \\
\hline Block copolymer (PLU) & $0,4-0,8 \%$ \\
\hline Riboflavin phosphete (RP) & $0.5 \%$ \\
\hline Water for injection q.s.p. & 100 \\
\hline
\end{tabular}

Table 3. Results of zeta potential and hydrodynamic radius of nanoemulsion riboflavin phosphate

\begin{tabular}{|l|c|c|}
\hline Samples (\%) & Zeta potential (mV) & Hydrodynamic radius (nm) \\
\hline CMT 5 + PLU 0,4 & 36.40 & 41.50 \\
\hline CMT 10 + PLU 0,4 & 32.56 & 41.90 \\
\hline CMT 5 + PLU 0,8 & 19.30 & 35.76 \\
\hline CMT 10 + PLU 0,8 & 18.80 & 35.81 \\
\hline
\end{tabular}

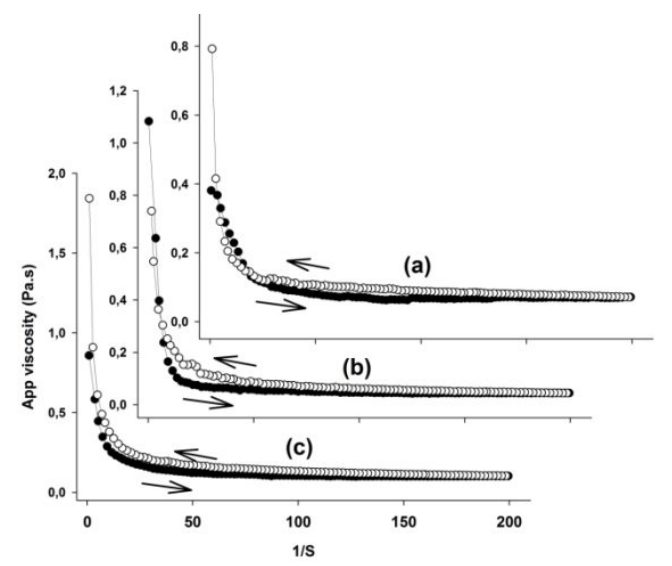

Figure 14. Rheological properties of nanoemulsion riboflavin phosphate. (a) Empty nanoemulsion. (b) nanoemulsion with 5\% of CMT. (c) nanoemulsion with $10 \%$ of CMT.

\section{CONCLUSION}

The present work showed the implementation of a controlled UVA LED based optical system device and the formulation of a new riboflavin. Equipment and drug are designed to treat especially Keratoconus disease on corneal tissue, the first lens of human eye, by a process called cross-linking. The equipment aimed at accelerating the treatment time by increasing the power density and keeping the well-known energy dose of $5.4 \mathrm{~J} / \mathrm{cm}^{2}$ by reducing exposure time. Additionally, riboflavin is incorporated in a system with nanometric dimensions, nanoemulsion, which combines the properties of bioadhesion and permeability increasing, so as to allow the riboflavin permeate through the corneal epidermis and to be kept in the stroma. This procedure tends to be faster, it has less infection risk, it is a painless procedure and offers a quick recovery to the patient.

The optical system developed proved to be very efficient in delivering a homogeneous power distribution on focus plane. The variations observed are less then $\pm 10 \%$ of average flux power, which complies with laser and high power LED standard IEC 60601-2-22. Therefore, the entire treating corneal area will receive the same radiation, resulting in equally collagen fibers bonds enlargement, which arise in a better treatment.

The power control system showed to be very efficient in controlling the LED output power. It has achieved the output power specification of $45 \mathrm{~mW} / \mathrm{cm}^{2}$ at a current below LED limit. The architecture of hardware and software controllers 
in cascade resulted in increased output power velocity readings, pulse operation in steady-state regime, without overshoot and fast responses. When the UV light output reaches stability, the steady-state error is less than $10 \%$. This rise-time value of 30 milliseconds, in worst case, denotes an insignificant loss of energy during entire treatment that takes minutes, and is perfectly acceptable by laser standard.

The device prototype had excellent performance to guarantee the mechanical distances between the lens involved and stability during the use. Additionally it has a portable and light design, which makes the transportation and set up easy to end user.

The riboflavin-nanostructured was developed and the first quality parameters were measured like zeta potential and rheological parameters. The results showed that the nanoemultion will have good bioadhesion to corneal surface and that it is ready to start the studies in rabbit's corneas. These studies will be carried out in partnership with Unifesp (Universidade Federal de São Paulo), which will receive one XLink prototype and samples of riboflavin to advance with riboflavin development.

\section{ACKNOWLEDGMENT}

The authors thank Opto Eletrônica S.A. and the Brazilian research and project financing FAPESP, for the financial support for this project.

\section{REFERENCES}

[1] Wollensak, G., Spoerl, E. and Seiler, T., "Riboflavin/ultraviolet-a-induced collagen crosslinking for the treatment of keratoconus," American Journal of Ophthalmology 135(5), 620-627 (2003).

[2] Schirmbeck, T., Paula, J. S., Taranta Martin, L. F., Crósio Filho, H., and Romão, E., "Eficácia e baixo custo no tratamento do ceratocone com o uso de lentes de contato rígidas gás-permeáveis," Arq Bras Oftalmol 68 (2), 219222(2005).

[3] Chew, J., "Strengthen cornea in 11 minutes," The Straits Times, May 03, 2012.

[4] Krueger, Ronald R., Spoerl, E. and Herekar, S., "Rapid vs standard collagen CXL with equivalent energy dosing," Proc. 3rd International Congress of Corneal Collagen Cross-Linking, (2007).

[5] Jaycock, P.D., Lobo, L., Ibrahim, J., Tyrer, J. and Marshall, J., "Interferometric technique to measure biomechanical changes in the cornea induced by refractive surgery," Cataract \& Refract Surgery 31(1), 175-184(2005).

[6] Sliney, D.H., and Wolbarsht, M.L., "Safety with LASERS and other optical sources", Plenum Publishing Corp, New York, (1980).

[7] Avedro Inc, "Implications of New Absorption, Diffusion and Scattering Coefficients for Corneal Cross-linking with Riboflavin", Clinical update \& Reserch news 1, 1(2012).

[8] Kanellopoulos A.J., "Long term results of a prospective randomized bilateral eye comparison trial of higher fluence, shorter duration ultraviolet A radiation, and riboflavin collagen cross linking for progressive keratoconus," Clinical Ophthalmology 6, 97-101(2012).

[9] Touboul D., Efron N., Smadja D., Praud D., Malet F. and Colin J., "Corneal confocal microscopy following conventional, transepithelial, and accelerated corneal collagen cross-linking procedures for keratoconus," Refractive Surgery 28(11), 769-776(2012).

[10] Gatzioufas Z., Richoz O., Brugnoli E. and Hafezi F., "Safety profile of high-fluence corneal collagen cross-linking for progressive keratoconus: preliminary results from a prospective cohort study", Refractive Surgery 29(12), 846848(2013).

[11] Trattler, W., "Corneal crosslinking for Keratoconus and LASIK complications," All about vision, May 2015,<http://www.allaboutvision.com/conditions/corneal-crosslinking.htm> (10 July 2015).

[12] Dorf R.C., [Modern Control Systems], 9th Ed. Prentice-Hall, 174-370 (2001).

[13] ELLIS, G., [Control System Design Guide], 3rd Ed. Elsevier, (2004).

[14] Sedra A. S. and Smith, K. C., [Microeletrônica], 5th Ed. Pearson Prentice-Hall, 141-579(2007).

[15] Mota, A. D. and Paiva, M. S. V., "Design of micro-second pulsed laser mode for ophthalmological CW Self-Raman laser," Proc. Spie Photonics West. Vol. 7912, 79121Y1 (2011).

[16] Bottos, K.M., Schor P., Dreyfuss J.L., Nader H.B. and Chamon W., "Effect of corneal epithelium on ultraviolet-A and riboflavin absorption," Arq Bras Oftalmologia 749, 348-351(2009). 
[17] Bottos, K.M., Dreyfuss J.L., Regatieri C.V. et al, "Immunofluorescence confocal microscopy of porcine corneas following collagen cross-linking treatment with riboflavin and ultraviolet A," Refractive Surgery 24, 715-719 (2008).

[18] Kymionis, G.D., Bouzoukis, D.I., Diakonis, V.F., Portaliou, D.M., Pallikaris, A.I. and Yoo, S.H., "Diffuse lamellar keratitis after corneal crosslinking in a patient with post-laser in situ keratomileusis corneal ectasia," Cataract Refractive Surgery 33, 2135-2137 (2007).

[19] Kymionis, G.D., Portaliou, D.M., Bouzoukis, D.I., et al., "Herpetic keratitis with iritis after corneal crosslinking with riboflavin and ultraviolet A for keratoconus," Cataract \& Refractive Surgery 33(11), 1982-1984(2007).

[20]Zamora, K.V. and Males, J.J., "Polymicrobial keratitis after a collagen cross-linking procedure with postoperative use of a contact lens: a case report," Cornea 28(4), 474-476(2009).

[21] Bottos, K.M., Oliveira, A.G., Bersametti, P.A., Nogueira, R.F., Lima-Filho, A.A., Schor, P. and Chamon, W., "Corneal absorption of a new riboflavin-nanostructured system for transepithelial collagen cross-linking," Ophthalmology, (2012).

[22]Nichia Corporation, "Specifications for UV LED", 07 July 2015, $<$ http://www.nichia.co.jp/specification/products/led/NCSU033B-E.pdf $>$.

[23] Malvern, "Zeta potential”, 07 July 2015, <http://www.malvern.com/en/products/measurement-type/zeta-potential/>.

[24] Malvern, "Kinexus Range”, 07 July 2015, <http://www.malvern.com/en/products/product-range/kinexus-range/> . 